\title{
Numerical Investigation on the Projectile with Base Cavity
}

\author{
Yang $\mathrm{Ni}^{\mathrm{a}}$, Zhihong $\mathrm{Ye}^{\mathrm{b}}$ and Haibo $\mathrm{Lu}^{\mathrm{c}}$ * \\ Artillery Military Theory Innovation and Operation Experiment Center, Army Academy of Artillery and \\ Air Defense, Nanjing 211132, China \\ atougaoy@126.com, bsubmission621@163.com, clhbboo@sohu.com
}

Keywords: Projectile with base cavity; Cavity shape; Aerodynamic drag; Boattail; CFD

\begin{abstract}
Base cavity plays a key role in reducing the aerodynamic drag of the projectile. In the paper, two kinds of base cavity shape for projectile are simulated by using axisymmetric Navier-Stokes equations. Under the supersonic flow condition, the distribution of the flow field parameters and the aerodynamic drag of the projectile were obtained. The numerical result shows that, in the paper, the shape of base cavity has little effect on the flow field of the projectile. But compare with the column cavity, the truncated-cone cavity shape reduces the aerodynamic drag of the projectile.
\end{abstract}

\section{Introduction}

The base drag make up 30 40\% of the total aerodynamic drag [1] for a common rotary projectile. Weakening the base drag is an effective way to reduce the aerodynamic drag of the projectile and increase the fire range of the artillery. Many investigations have been done in this field [2-9]. Base bleed, boattail and base cavity are all proved to be effective. Under the same flow condition, the range of fire for projectile with base cavity is further than the projectile without base cavity 3 5\% [10].

In this paper, two kinds of base cavity shape for projectile with boattail is investigated with numerical method. By the comparison between simulation results, flow fields and aerodynamic drag, the effect of the base cavity shape was discussed.

\section{Shape of Base Cavity}

In Fig.1, the schematic map of the projectile with different shape base cavity is shown. The difference between two cavity shape is that cavity of shape " $\mathrm{A}$ " is truncated-cone and shape " $\mathrm{B}$ " is a column one.

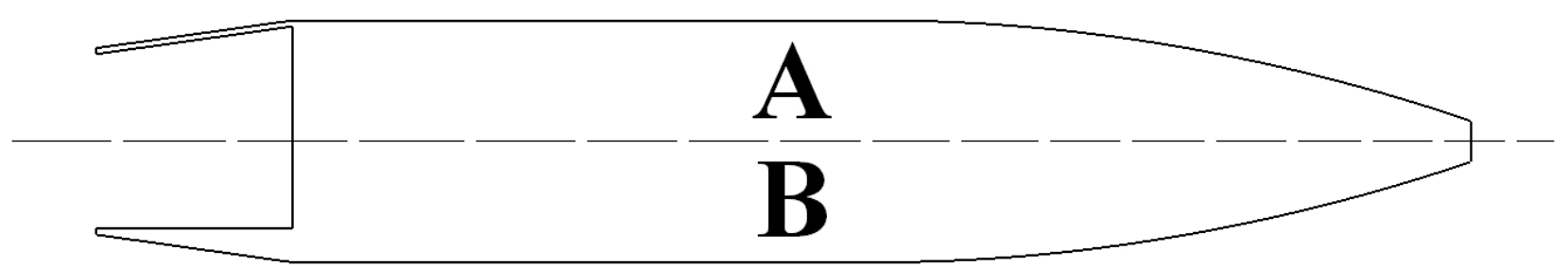

Figure 1. Schematic of the projectile shape

\section{Numerical Method}

Governing Equations The axisymmetric Navier-Stokes equation is given by [11]

$$
\partial U / \partial T^{+} \partial F(U) / \partial x+\partial G(U) / \partial r+1 / r \cdot \partial H(U) / \partial r=1 / \operatorname{Re} \cdot\left(\partial F_{v}(U) / \partial x+{ } G_{v}(U) / \partial r+1 / r \cdot \partial H_{v}(U) / \partial r\right)
$$

where $U$ is the conservation variable, $F, G$ and $H$ are the inviscid terms, $F_{v}, G_{v}$ and $H_{v}$ are the viscous terms. The $k$ - $\varepsilon$ turbulence model [12] is used in the simulation.

The convective terms are approximated by the Van Leer [13] splitting method and the central difference method is used for the viscous terms. For the time integration, the LU-SSOR scheme is used. 
Grid and Boundary Condition As shown in Fig. 2, the body-fitted grid (Shape "A") which is used in the simulation is given.

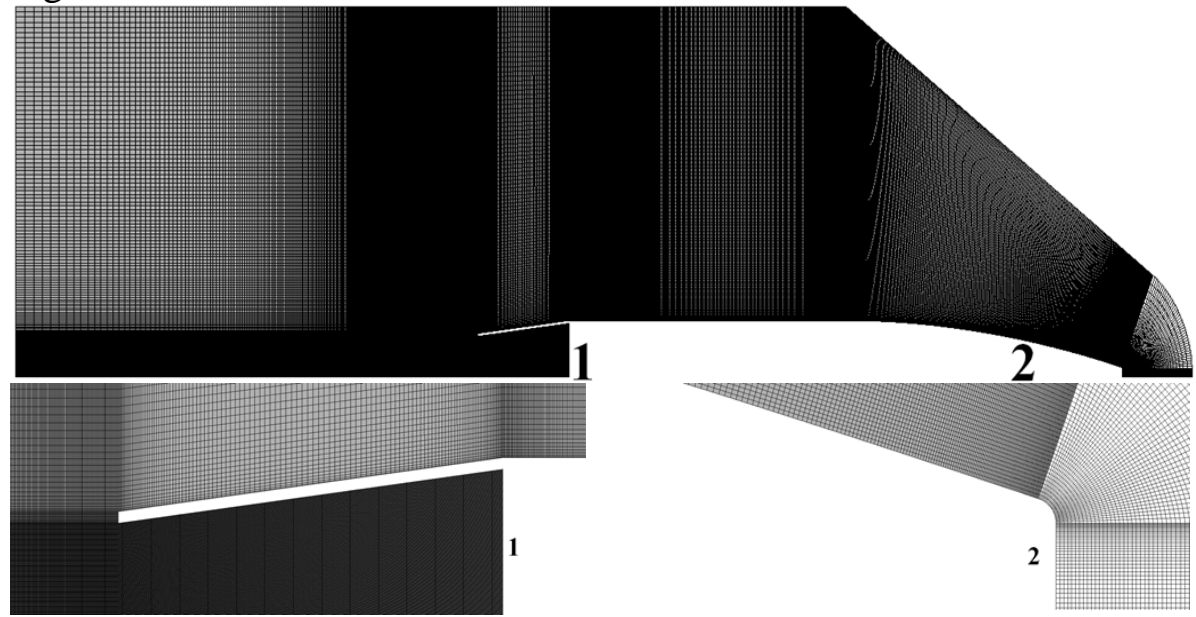

Figure 2. Grid of the simulation model (Shape "A")

The flow conditions are given in Table 1. The wall boundary condition is assumed to be no-slip and adiabatic.

Table 1 Boundary conditions

\begin{tabular}{|c|c|c|}
\hline Free stream parameter & Unit & Value \\
\hline Mach Number $(M a)$ & ---- & 1.97 \\
\hline Pressure $\left(p_{\infty}\right)$ & $\mathrm{Pa}$ & 101325 \\
\hline Temperature $\left(T_{\infty}\right)$ & $\mathrm{K}$ & 300 \\
\hline
\end{tabular}

\section{Results and discussion}

Flow field The distribution of flow field parameters is shown in Fig. 3- Fig. 6. As shown, a classical circumferential motion is formed around the projectile. A detached bow shock wave locates in front of projectiles and expansion wave form along the boattail (Fig. 3). The distribution of temperature is shown in Fig. 4, a local high temperature region was formed inside the cavity. The distribution of pressure is given in Fig. 5.

In Fig. 6, a locally enlarged $M a$ and streamline image of the base cavity region is given. As shown, a large circumfluence is formed inside the cavity.

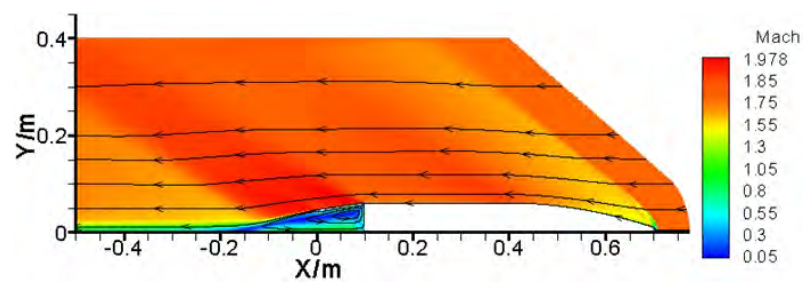

Shape A

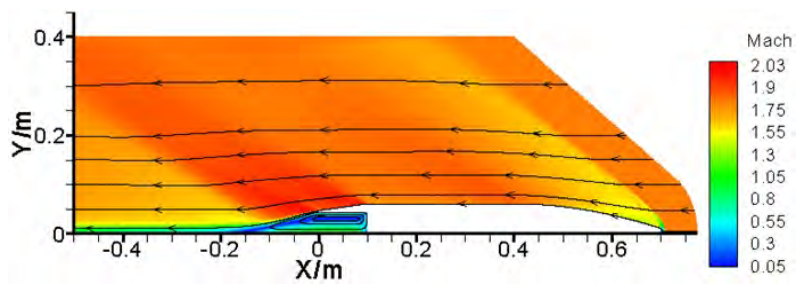

Shape B

Figure 3. Distributions of the Ma contours and the streamlines

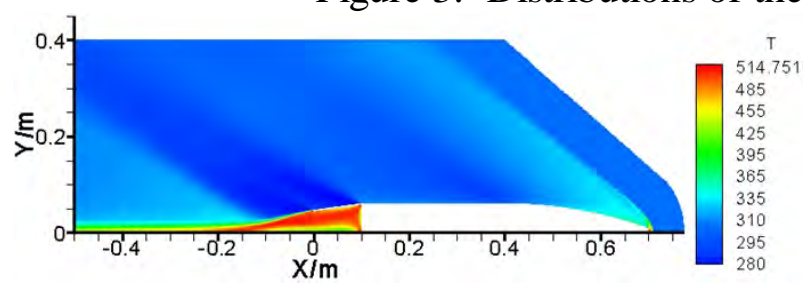

Shape A

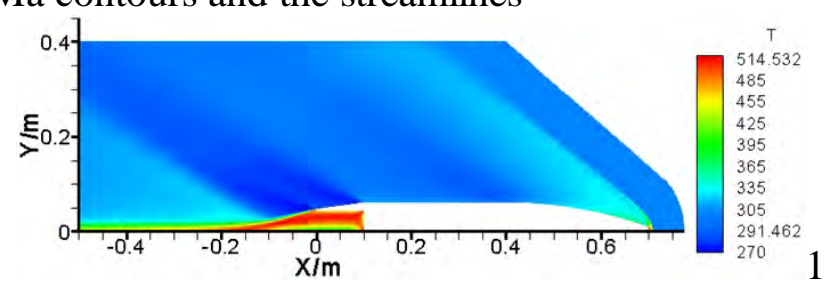

Shape B 
Figure 4. Distributions of the Temperature
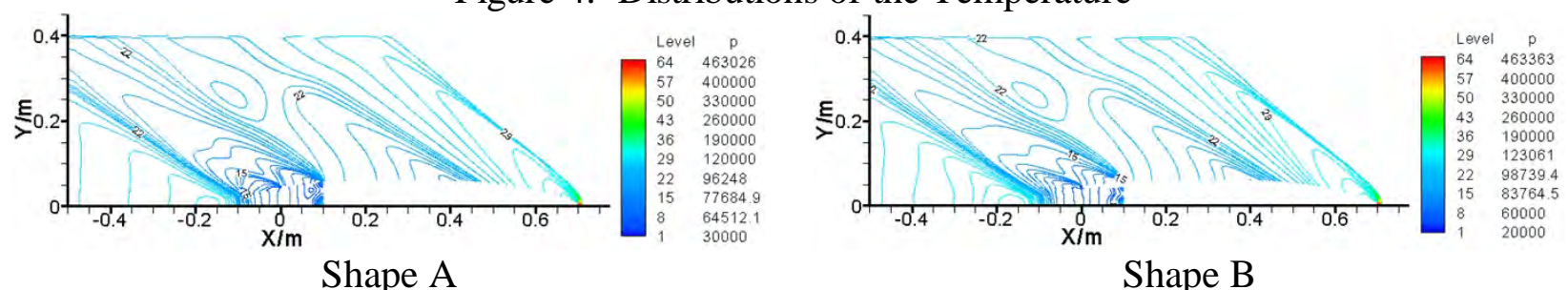

Figure 5. Distributions of the pressure

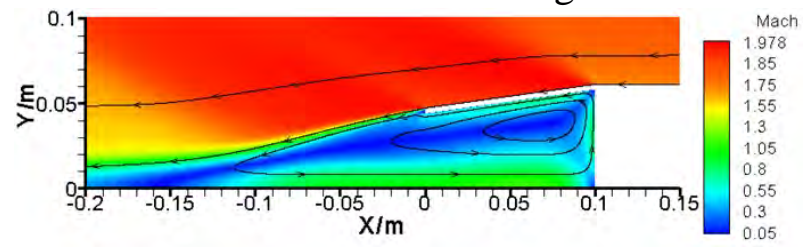

Shape A

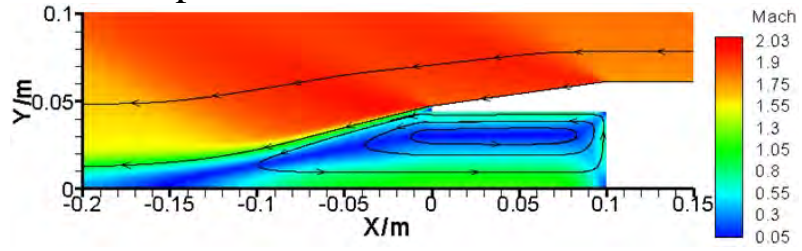

Shape B

Figure 6. Local enlarged drawing of the Ma-streamline distribution

Aerodynamic drag The drag coefficient $\left(C_{d}\right)$ of the projectile is given by the expression:

$$
C_{d}=F_{d} /\left(\frac{1}{2} \rho_{\infty} u_{\infty}^{2} \cdot S_{\text {ref }}\right)
$$

where $F_{d}$ is the aerodynamic resistance, $\rho_{\infty}$ is the density of the free stream, $u_{\infty}$ is the velocity of the free stream, $S_{\text {ref }}$ is the reference area which is the cross section of the projectile.

The drag coefficient $\left(C_{d}\right)$ of two projectiles is given in Table 2. As shown, the projectile with the truncated-cone cavity (shape "A") gets the smaller drag coefficient. As shown in Fig. 7, the trends of the distributions of pressure along the base wall of the two projectiles are consistent. From the center of the base wall to its edge, there is a sharp drop after a slight rise, and then raise sharp again. The difference is that the base wall of shape " $A$ " is larger than shape "B" (Fig. 7, the region 2), so, the base pressure of projectile with base cavity "A" is bigger than projectile with base cavity " $\mathrm{B}$ ", and then, the shape "A" has smaller drag coefficient.

Table 2 drag coefficient $\left(C_{d}\right)$

\begin{tabular}{|c|c|c|}
\hline Shape & A & B \\
\hline drag coefficient $\left(C_{d}\right)$ & 0.2797 & 0.2840 \\
\hline
\end{tabular}




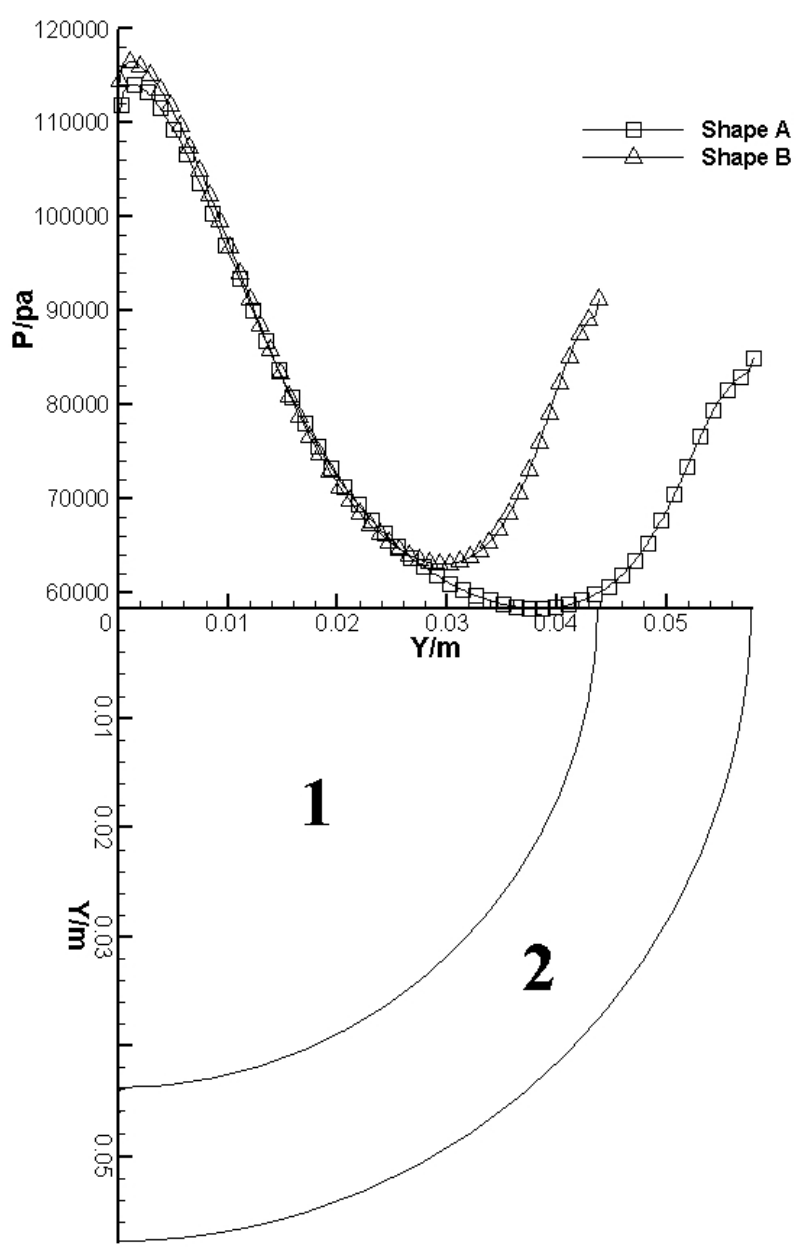

Figure 7. Pressure distributions on base wall and its acreage

\section{Conclusion}

Projectile with the different shape base cavity was investigated numerically in the paper. Compared with there flow field parameters distribution and the aerodynamic drag coefficient, the influence of the base cavity shape on the projectile was discussed. In the paper, the two kinds of base cavity shape has little effect on the trends of the pressure distributions along the base wall, but the truncated-cone shape base cavity causes to a larger base wall, this is benefit to increase the base pressure of the projectile and leads to a smaller aerodynamic drag.

\section{References}

[1] Sheng Zhongshu, Liu Yafei. Projectile Aerodynamics. Beijing: National Defense Industry Press, 1984. pp.36 (in Chinese)

[2] ZHUO Changfei, FENG Feng, WU Xiao song. Research on base flow field and aerodynamic characteristics of the supersonic base bleed projectile. Acta Aerodynamica Sinica, 32(6), Dec. 2014, pp. 783-790. (in Chinese)

[3] Chen X. H., Zhou Z. C., Huang H., et al. Numerical simulation of base bleed energy affecting aerodynamic performance of base bleed projectiles. Acta Armamentarii, 2010, 31(4): 448-452. (in Chinese)

[4] CHOR J. R., SHIN E., KIM C. K. Numerical study of base bleed projectile with external combustion. AIAA 2005-4352, 2005.

[5] Tan Junjie, Wang Fuhua, Xu Qin, Zhao Ruxiang. The Experimental Investigation and Analysis about the Effects of Tail Sting and Boat Tail on the Base Pressure of Projectiles at Supersonic Experiments and Measurements in Fluid Mechanics. 1997, 11(4): 13-17. (in Chinese) 
[6] Cao Jiayi, Lu Chuanjing, Chen Ying, Chen Xin, Li jie. Research on the base cavity of a sub-launched projectile. Journal of Hudrodynamics, Vol. 24, Apr. 2012, pp. 244-249.

[7] A. Ibrahim, A. Filippone, Supersonic aerodynamics of a projectile with slot cavities, Aeronautical Journal, 114(2010) 15-24.

[8] Cao Jiayi, Lu Chuanjing, Chen Ying, Chen Xin, Li jie, Research on the base cavity of a sub-launched projectile, Journal of Hudrodynamics, 24(2012) 244-249.

[9] Howell Jeff, Sims-Williams Daid, Sprot Adam, Hamlin Fred, Dominy Robert, Bluff body drag reduction with ventilated base cavities, SAE Technical Papers, 5(2012) 152-160.

[10] Wei Huizhi, Zhu Hesong. The design theory of projectile. Beijing: National Defense Industry Press, 1985. (in Chinese) pp.36

[11] Wu Xiaosong. The numerical investigation on flow field of projectile (with base bleed). Journal of ballistics, 4 (3), pp. 39-43, 1992 (in Chinese)

[12] Tao Wenquan. Numerical Heat Transfer, 2nd edn. Xi' an: Xi' an Jiaotong University Press, 2001 (in Chinese)

[13]Wang Xuede, Tan Junjie, Lin Xiaohong, Tang Zhenhua. Research on parallel numerical simulation of N-S equations based on Van Leer+AUSM scheme. Jounal of Astronautics, Vol. 31, Apr. 2010, pp. 986-992. (in Chinese) 